\title{
Bi-objective robust optimization of machined surface quality and productivity under vibrations limitation
}

\author{
M.A. Sahali ${ }^{1, a}$, R. Serra ${ }^{2, b}$, I. Belaidi ${ }^{1, c}$ and H. Chibane ${ }^{2, d}$ \\ ${ }^{1} \mathrm{UMBB}$, Equipe de recherche en mécanique et ingénierie des systèmes et procédés, Laboratoire d'Energétique, \\ Mécanique et Ingénieries, Université M’hamed Bougara de Boumerdes 35000, Algerie \\ 2 INSA Centre Val de Loire, Université François Rabelais, Laboratoire de Mécanique et Rhéologie - CEROC, 3 rue de la \\ chocolaterie CS 23410, 41034 Blois Cedex, France
}

\begin{abstract}
In this contribution, a bi-objective robust optimization of cutting parameters, with the taking into account uncertainties inherent in the tool wear and the tool deflection for a turning operation is presented. In a first step, we proceed to the construction of substitution models that connect the cutting parameters to the variables of interest based on design of experiments. Our two objectives are the best machined surface quality and the maximum productivity under consideration of limitations related to the vibrations and the range of the three cutting parameters. Then, using the developed genetic algorithm that based on a robust evaluation mechanism of chromosomes by Monte-Carlo simulations, the influence and interest of the uncertainties integration in the machining optimization is demonstrated. After comparing the classical and robust Pareto fronts, A surface quality less efficient but robust can be obtained with the consideration of uncontrollable factors or uncertainties unlike that provides the deterministic and classical optimization for the same values of productivity.
\end{abstract}

\section{Introduction}

In machining by material removal, some variability due to geometric, kinematic, thermal, and mechanical errors (deflection of the cutting tool) are a source of generating geometric irregularities [1-3] affecting randomly the quality of the finite piece. Taking into account these uncertainties in the control procedures and optimization of cutting conditions is therefore of great interest for a better mastery of productivity and of the desired quality of the pieces.

In this present paper, we focus mainly on errors due to deflection and tool wear. The error rate generated by the tool deflection in the conventional machining is approximately $44 \%$ of the estimated total error including positioning of the machine, thermal elongation, the trajectory of the piece and tool and the alignment of the piece [4]. The uncertainties in this context may exceed 40 microns in finishing [5] and the surface degradation caused by the tool deflection is even more important during operations requiring slender and thin cutting tools of best maneuverability (milling of molds). Indeed, the error on the surface quality caused by the tool deflection can attaining 100 microns during the milling of a hardened steel mold with a ball end mill in cemented carbide [6], what has also motivated the development of models to estimate the geometric errors and the implementation of compensation algorithms in numerical control in [7] and

\footnotetext{
a e-mail: Akli_Sahali@hotmail.fr

b e-mail: roger.serra@insa-cvl.fr

$c$ e-mail: idir-belaidi@umbb.dz

d e-mail: hicham.chibane@univ-tours.fr
}

the mathematical formulation of the error related to the machine tool in [2].

The nose abrasive wear of the cutting edge is systematic and inevitable. It is more significant when using HSS tools. Exceptionally, in turning at high speed the profile of the cutting edge is quickly changed due to friction and high temperature between the workpiece and the flank face of tool. The wear widens the nose radius and the feed is decreased gradually with time. This has a positive influence on the roughness of the machined surface [8].

In this context, we have proposed an optimization of cutting parameters that realize a compromise between a minimum roughness of the machined surface and maximum productivity. The uncertainties inherent to wear and tool deflection for longitudinal turning operation are taken into account. To relate theory and practice, we have built models of substitution by multiple linear regressions from design of experiments for the prediction and optimization of cutting parameters. Using the developed genetic algorithm [9] that based on a robust evaluation mechanism of chromosomes by Monte-Carlo simulations, the influence and interest of the uncertainties integration in the machining optimization will be demonstrated.

\section{Robust optimization}

The economical and reliable machining is the result of a robust optimization of machining operative conditions leading to less sensitive solutions to variabilities. In other words this is an optimization taking into account the uncertainties of cutting parameters [10].

This is an Open Access article distributed under the terms of the Creative Commons Attribution License 2.0, which permits unrestricted use, distribution, and reproduction in any medium, provided the original work is properly cited. 
The robust optimization procedure requires making the stochastic problem in deterministic state. Indeed, the introduction of uncertainty in the input parameters under the probability laws makes the problem in stochastic state, because the output parameters become random by propagation of errors. Therefore to bring it to a deterministic problem it must measure the robustness of the dependent variables by calculating the expectation, variance or both simultaneously [11-14]. At a point $x$ affected by uncertainty, the expectation measurement of a function $f(x)$ is the average value estimation of this function in the entourage of the studied point $x$; However, the "expectation" function performs a smoothing of the original "objective" function. The obtained solution after an optimization by the expectation is called efficient-robust solution.

In addition, the robustness measurement by the calculation of variance considers the dispersion of function around the studied point, and the minimization of the variance provides information on a purely robust solution against objectives [15]. The formulation of the robust optimization problem is performed in different ways. The original "objective" function can be replaced by its expectation and the original "constraint" function can be added to its variance [16]. A combination of pure robustness and efficiency by substituting the classical "objective" function by an aggregation of mean-variance with a weighting factor is also feasible [17]. Treating the expectation of the original functions and consider its variance as a constraint (M-robustness) and the reverse (V-robustness) is another possible solution [18].

\section{Robust optimization in machining}

Sun et al. [19] have conducted a robust multi-objective optimization by particle swarm method in the field of metal forming; the objective and constraint functions are obtained by a design of numerical experiments. In addition, they used the six-sigma principle to assess the variations of stress caused by disturbances of the decision variables. Similarly, through the computerassisted manufacturing (CAM), Jeang [20] was able to obtain a robust substitution model by linking the machining time and the uncertainties of decision variables using the design of experiences methodology and response surface, implemented under the CATIA ${ }^{\mathrm{TM}}$ software. The minimization of the machining time under uncertainty, taking into account the quality of the machined surface, has opened new perspectives.

Landers et al. [21] have proposed a strategy for robust control of the cutting force, in order to compensate the cutting process submitted to variability. Ivester and Danai [22] have suggested the adaptive control optimization, where the machine settings are adjusted according to the cutting process reaction and in a manner to compensate inaccuracies in the milling of thin walls. Huang et al. [23] have used the finite element analysis to predict the error due to the tool deflection and system instability by a thermo-mechanical model, and thanks to min-max method, they have realized a robust optimization of cutting parameters. Paiva et al. [24] for a hard steel

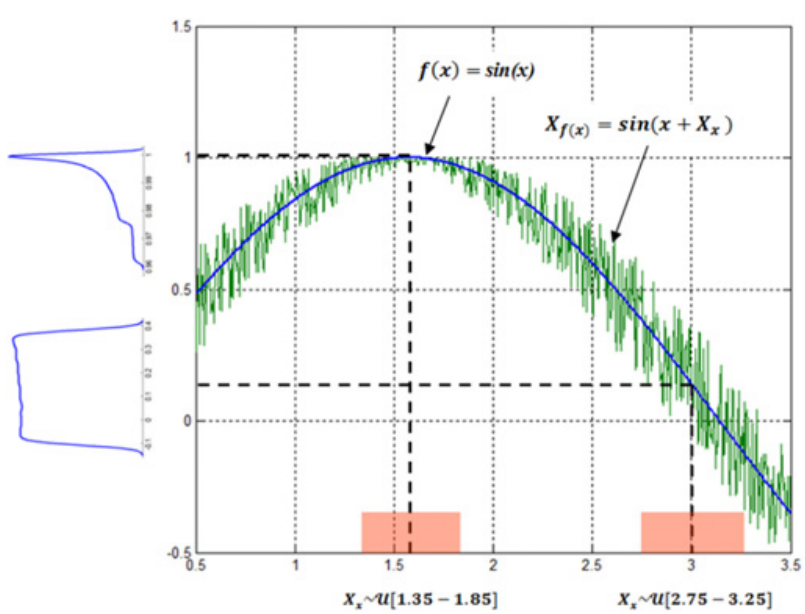

Figure 1. Estimate of the probability density of the random variable $f\left(x+X_{x}\right)$ in green, at two different points by the Monte Carlo simulations.

turning have studied the influence of cutting parameters $\left(V, f, a_{p}\right)$ and two uncertain parameters (tool wear, piece hardness) on the surface roughness by design of experiences methodology and then proceed to the robust optimization with substitution models.

\section{Formulation of a robust optimization problem}

If a decision variable $x$ is uncertain and its distribution follows a uniform law $X_{x} \approx U[-\delta, \delta]$, thus the original function $f(x)$ in turn becomes a random variable $X_{f(x)}$ by uncertainty propagation whose probability distribution is unknown a priori for each point. The classical optimization problem becomes stochastic; it must be reduced to a deterministic problem with the robustness measurement denoted as $\varphi\left(f\left(x+X_{x}\right)\right)$ (Eq. (3)). The most used robustness measurement is mean-variance aggregation (Eq. (3)) calculated with the Monte Carlo simulations (Fig. 1):

We can formulate the classical and robust optimization problems respectively in (Eq. (1)) and (Eq. (2)) as follows:

$$
\begin{aligned}
& \left\{\begin{array}{c}
\text { minimize } f(x) \\
\text { such as } g_{j}(\boldsymbol{x} \leq 0 \quad j=1, \ldots, n \\
x_{\min } \leq \boldsymbol{x} \leq x_{\max }
\end{array}\right. \\
& \left\{\begin{array}{c}
\text { minimize } \varphi\left(f\left(\boldsymbol{x}+X_{x}\right)\right) \\
\text { such as } g_{r_{j}}(\boldsymbol{x}) \leq 0 \quad j=1, \ldots, n \\
x_{\min } \leq \boldsymbol{x} \pm \delta x \leq x_{\max }
\end{array}\right.
\end{aligned}
$$

where $\delta x$ is the half uncertainty interval (tolerance) of design variables. And $\boldsymbol{x}$ is the design variable vector, $x_{\text {min }}$ and $x_{\max }$ are the lower and upper bounds of the search space. $\varphi\left(f\left(x+X_{x}\right)\right)$ is the robust "objective" function that replaces the classical objective function $f(\boldsymbol{x})$ and $g_{r j}(\boldsymbol{x})$ are the n robust "constraint" functions (explicated 


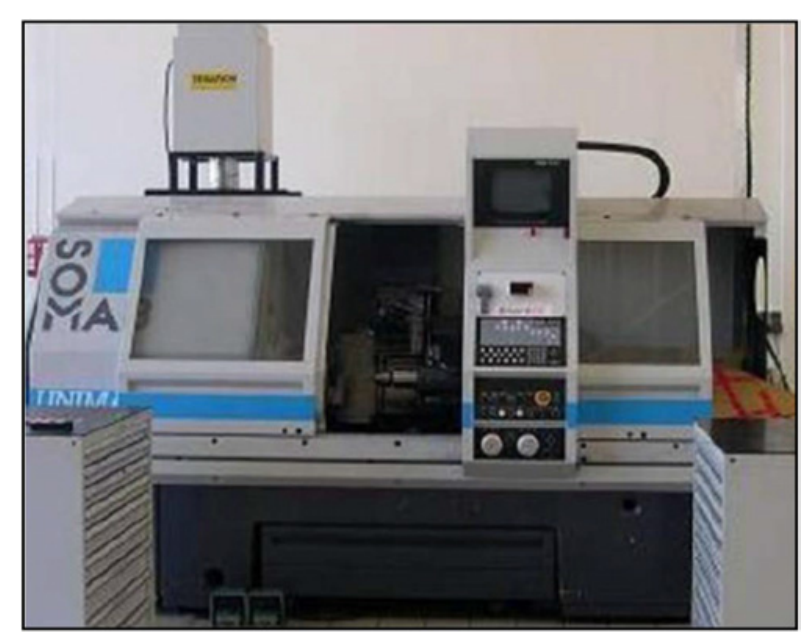

Figure 2. NC Turning lathe SOMAB 500.

in Eq. (4)) which also substitute the classical "constraint" $g_{j}(\boldsymbol{x})$.

$$
\begin{aligned}
\varphi\left(f\left(\boldsymbol{x}+X_{x}\right)\right)= & \alpha * E\left[f\left(\boldsymbol{x}+X_{x}\right)\right] \\
& +(1-\alpha) * \sigma\left(f\left(\boldsymbol{x}+X_{x}\right) .\right.
\end{aligned}
$$

With $\alpha$ is a weighting factor. $0 \leq \alpha \leq 1$,

$\alpha=0$ : the solution is called purely robust,

$\alpha=1$ : the solution is called efficient and robust,

$E:$ is the expectation of the objective function,

$\sigma:$ is the standard deviation of the objective function,

In our case, the standard deviation measurement is taken to respect the boundaries of the new search space defined by the following news reliable constraints [10]:

$$
g_{r_{j}}(\boldsymbol{x})=g_{j}(\boldsymbol{x})+\beta * \sum_{i=1}^{n}\left|\frac{\partial g(\boldsymbol{x})_{j}}{\partial x_{i}}\right| \Delta x_{i}
$$

$\left|\frac{\partial g(x)_{j}}{\partial x_{i}}\right|:$ is the gradient absolute value of the $j^{\text {th }}$ constraint relative to the $i^{\text {th }}$ design variable.

$\beta$ is a penalty factor or a confidence coefficient that controls the robustness degree.

\section{Case study in turning}

The longitudinal turning tests were performed on a turn SOMAB 500 with a power of $24 \mathrm{~kW}$ and having a maximum rotational speed of $2500 \mathrm{rev} / \mathrm{min}$ (Fig. 2). The chosen material is $100 \mathrm{C} 6$, an alloyed steel with strength of about $850 \mathrm{MPa}$ and a hardness of $217 \mathrm{HB}$. The insert geometry is ISO CNMG 120408 mounted on tool ISO DCLN2525M12.

To implement our optimization algorithm and to choose the optimum cutting conditions, several parameters to be measured are selected:

- Machined surface quality: we have measured the surface roughness $\mathrm{Ra}(\mu \mathrm{m})$ using a roughnessmeter.

- Productivity: the metal removal rate $\mathrm{Q}\left(\mathrm{cm}^{3} / \mathrm{min}\right)$ is calculated for each test.

$$
Q=1000 * V * f * a_{p} .
$$

- Vibration of the cutting tool: the signals coming from the triaxial accelerometer (4520) mounted on the tool are collected using a multi-analyzer 3560 Brüel and Kjær in three directions and recorded directly onto PC. The acquisition time is related to the used cutting speed (between $8 \mathrm{~s}$ and $24 \mathrm{~s}$ ) and the sampling frequency is $16384 \mathrm{~Hz}$.

- The accelerations are more important in the tangential direction $(\mathrm{z})$, hence the choice of this direction for our analysis.

\subsection{Multiple linear regression}

Using multiple linear regression and design of experiments, we can mount a predictive theoretical model of observed or measured values with very good accuracy $[25,26]$.

$$
y_{i}=\beta_{0}+\sum_{j=1}^{p} \beta_{j} X_{i j}+\varepsilon_{i}
$$

where $\varepsilon_{i}$ is the module error that expresses or summarize the missing information in the linear regression of the $y_{i}$ values. $X_{i 1}, \ldots, X_{i j}$ et $\beta_{0}, \ldots, \beta_{j}$ are the coefficients of parameters to be estimated. A series of statistical tests is conducted to judge the quality of the model:

- The global analysis of the system;

- The study of model coefficients;

- The analysis of residues;

Our design of experiments is factorial, orthogonal and complete; it contains 27 trials (Table 1) [27].

\subsection{The meta-models}

Based on the results of carried investigations, the linear regression and the analysis of variance (ANOVA) were used to determine the empirical models, and the influential factors for each output $\left(R a, A_{z}\right)$ as a function of the inputs $\left(a_{p}, f, V\right)$.

\section{Optimization}

Using meta-models mentioned above, we want to optimize the cutting parameters that achieve a compromise between maximum productivity and the minimal roughness of the machined surface under the variability of the depth of cut and tool feed. Thereafter, a comparison between the robust optima (under uncertainty) and efficient optima (classical) is processed.

For this, the developed genetic algorithm MC-GA is used for its efficiency to treat the optimization problems under uncertainty [9]. The proposed "robust" genetic algorithm contains the same steps as the "deterministic" genetic algorithm.

\subsection{The uncertain parameters}

In this section, we will consider the uncertainties of two parameters that have a significant and direct influence on the dependent variables. The two parameters are the 
Table 1. Design of experiments.

\begin{tabular}{|l|l|l|l|l|l|}
\hline $\mathbf{N}^{\circ}$ & $\boldsymbol{a}_{\mathbf{p}}$ & $\boldsymbol{f}$ & $\boldsymbol{V}$ & $\mathbf{R a}(\boldsymbol{\mu m})$ & $\mathbf{A z}$ \\
\hline 1 & 1 & 0.15 & 100 & 0.484 & 12.2509 \\
\hline 2 & 1 & 0.15 & 200 & 0.457 & 47.6386 \\
\hline 3 & 1 & 0.15 & 300 & 0.511 & 90.7654 \\
\hline 4 & 1 & 0.3 & 100 & 1.793 & 19.7482 \\
\hline 5 & 1 & 0.3 & 200 & 2.049 & 66.9501 \\
\hline 6 & 1 & 0.3 & 300 & 2.054 & 149.6158 \\
\hline 7 & 1 & 0.45 & 100 & 3.442 & 18.8094 \\
\hline 8 & 1 & 0.45 & 200 & 3.652 & 89.4253 \\
\hline 9 & 1 & 0.45 & 300 & 3.988 & 169.5976 \\
\hline 10 & 1.5 & 0.15 & 100 & 0.856 & 12.9179 \\
\hline 11 & 1.5 & 0.15 & 200 & 0.598 & 47.9771 \\
\hline 12 & 1.5 & 0.15 & 300 & 0.645 & 87.1003 \\
\hline 13 & 1.5 & 0.3 & 100 & 3.082 & 19.3565 \\
\hline 14 & 1.5 & 0.3 & 200 & 1.967 & 73.3288 \\
\hline 15 & 1.5 & 0.3 & 300 & 2.516 & 119.7132 \\
\hline 16 & 1.5 & 0.45 & 100 & 4.864 & 25.2293 \\
\hline 17 & 1.5 & 0.45 & 200 & 3.537 & 98.9062 \\
\hline 18 & 1.5 & 0.45 & 300 & 4.466 & 198.9357 \\
\hline 19 & 2 & 0.15 & 100 & 1.320 & 18.7534 \\
\hline 20 & 2 & 0.15 & 200 & 1.178 & 49.5799 \\
\hline 21 & 2 & 0.15 & 300 & 1.203 & 99.6932 \\
\hline 22 & 2 & 0.3 & 100 & 2.921 & 35.2407 \\
\hline 23 & 2 & 0.3 & 200 & 2.670 & 68.6314 \\
\hline 24 & 2 & 0.3 & 300 & 2.393 & 164.2688 \\
\hline 25 & 2 & 0.45 & 100 & 6.549 & 51.738 \\
\hline 26 & 2 & 0.45 & 200 & 5.472 & 157.2322 \\
\hline 27 & 2 & 0.45 & 300 & 5.811 & 347.1723 \\
\hline & & & & & \\
\hline
\end{tabular}

Table 2. The regression models for each output with their respective adjusted coefficients of determination.

\begin{tabular}{|c|c|}
\hline Empirical models & $\mathbf{R}^{2} \mathbf{a j}$ \\
\hline$R a=-0.85-0.43 * a_{p}+12.08 * f-8.07 * 10^{-3} *$ & \\
$V+1.37 * a_{p} * f+5.25 * 10^{-3} * V * a_{p}-9.52 *$ & 0.99 \\
$10^{-5} * f * \mathrm{~V}$ & \\
$(8)$ & \\
\hline$A_{z}=$ & \\
$173.48-114.58 * a_{p}-660.75 * f-0.51 * \mathrm{~V}+$ & \\
$325.12 * a_{p} * f+0.36 * a_{p} * V+2.38 * f * \mathrm{~V}$ & 0.98 \\
$(9)$ & \\
\hline
\end{tabular}

tool feed $(f)$ and depth of cut $\left(a_{p}\right)$. Their variabilities are assumed to follow a uniform distribution. Due to the wear of the cutting edge, the feed pitch and the thickness of the chip decrease progressively along the machined length (systematic dispersion on Fig. 3). Its uncertainty is estimated through cutting tool flank wear VB according to ISO 3685 Standard (1993) [28]. This wear type causes geometrical irregularities of first order (cylindricity, planarity...), but increasingly a better machined surface quality is obtained due to the systematic reduction of the feed.

$$
V B_{n}=V B * \tan \alpha
$$

$\alpha$ : angle of flank in orthogonal section.

The radial deflection of tool $\left(D_{r}\right)$ caused by the repulsive cutting effort (Fig. 4) generates a random dispersion of the machined profile height (defects of second order). Also, the decline of the cutting tip $\left(U r_{\varepsilon}\right)$
Table 3. The parameters value of used genetic algorithm.

\begin{tabular}{|l|l|}
\hline Dimension of population & 200 \\
\hline Number of Monte-Carlo simple & 50000 \\
\hline Selection & by tournament \\
\hline Rate of crossover & 0.8 \\
\hline Rate of mutation & 0.01 \\
\hline Number of generation & 200 \\
\hline
\end{tabular}

resulted from the wear of nose radius (Figs. 3-4) is the cause of geometrical irregularities of first order.

The beginning of wear can lead to change the nose radius in the sense of an equivalent radius greater than nominal radius thus to temporarily improve the machined surface quality [8]. Consequently, the uncertainty about the depth of cut $a_{p}$ is calculated by the sum of the last two uncertainties $\left(U a_{p}=U r_{\varepsilon}+D_{r}\right)$ and it is estimated at about $10 \%$.

In the formulation of our problem under uncertainty, the classical "objective" functions (R and $\mathrm{Q})$ that have become random $\left(\bar{R}_{a}\right.$ and $\left.\bar{Q}\right)$, are replaced by their expectations $\left(E\left(\bar{R}_{a}\right)\right.$ and $E(\bar{Q})$. In addition, the classical "constraint" functions are added to the standard deviation of stochastic "constraint" functions $\sigma\left(\bar{A}_{z}\right)$ multiplied by a penalty coefficient $\beta$ to form new robust constraints (Eq. (8)).

To reduce geometric and dimensional defects of first order, a maximum level of vibration $A_{\mathrm{z} \max }$ was fixed according with the obtained results during a previous optimization campaign [27].

$$
\left\{\begin{array}{c}
\text { minimize } E\left(\bar{R}_{a}\right) \\
\text { minimize } E(\bar{Q}) \\
\text { such as } \\
A_{2}+\beta * \sigma\left(\bar{A}_{z}\right) \leq A_{z} \text { max } \\
100 \leq V \leq 300 \\
0.15 \leq f \leq 0.45 \\
1 \leq a_{p} \leq 2 \\
\text { with } \\
A_{z} \text { max }=200 \mathrm{~m} / \mathrm{s}^{2} \\
\tilde{f}=U\left[f-V B_{n} ; f\right] \\
\tilde{a}_{p}=U\left[a_{p}-\left(a_{p} * U a_{p}\right) ; a_{p}\right] \\
\beta
\end{array}\right.
$$

\section{Results and discussion}

For depth of cut $a_{p}$ of $2 \mathrm{~mm}$, the "classical" feasible domain is defined by the maximum vibration level (blue on Fig. 6), by the maximum and minimum allowable advances [0.15-0.45] (black on Fig. 6). On the other hand, the "robust" feasible domain is bounded by the noise of vibration function (green) and by the noise functions of maximum and minimum permitted feed (respectively: dark green and red on Fig. 6).

By analyzing the curves in Fig. 6, it is essential to take the maximum values of feed $f$ and the minimum cutting speed $V$ to respect the threshold acceleration 


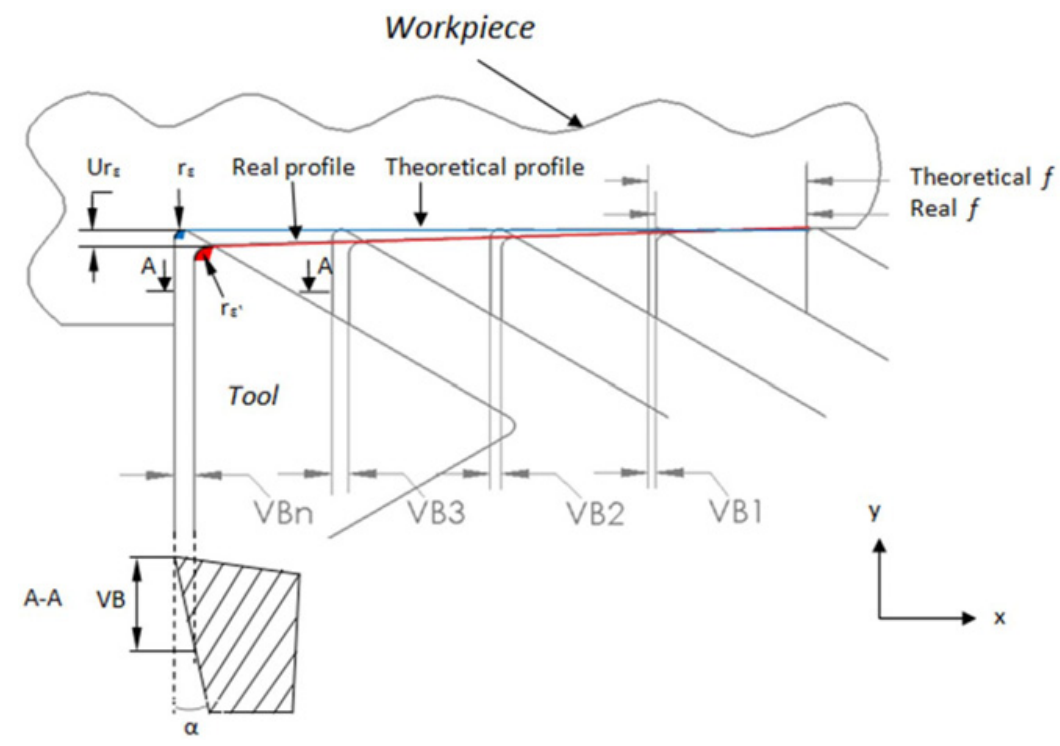

Figure 3. The uncertainty of tool feed caused by flank wear VB of the cutting tool in turning.

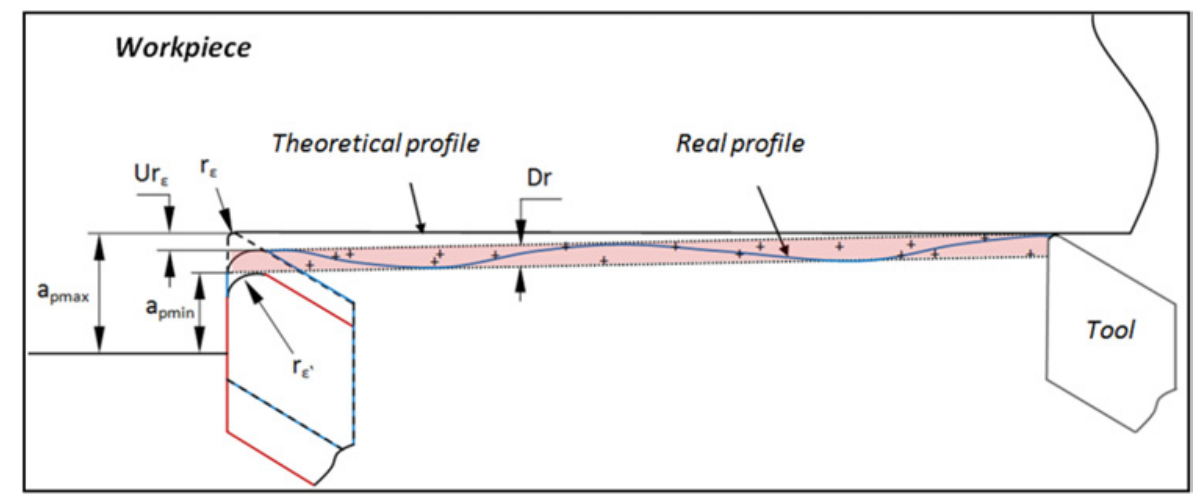

Figure 4. The uncertainty of depth of cut caused by the tool deflection and wear of nose radius.

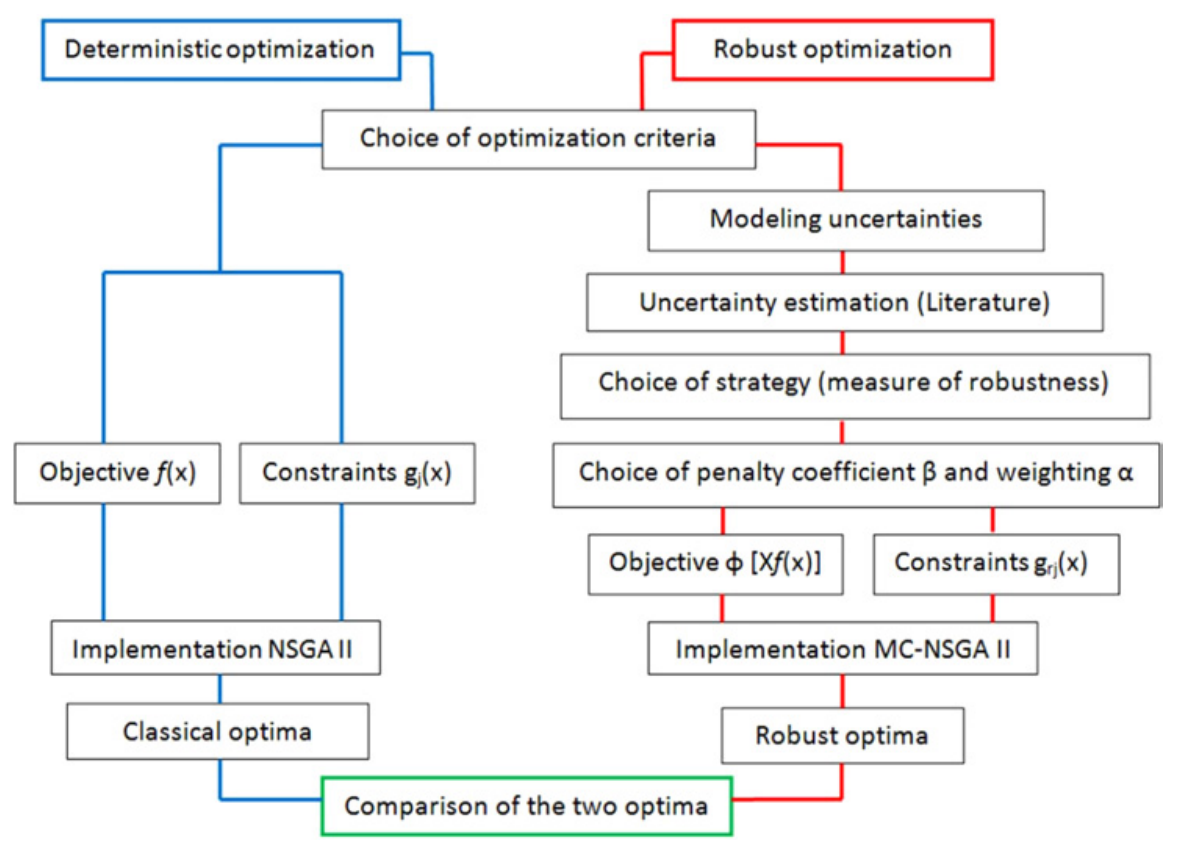

Figure 5. Organogram of classical and robust optimization. 
Table 4. Comparison of efficient and robust optima.

\begin{tabular}{|l|c|c|c|c|c|c|c|c|c|}
\cline { 2 - 9 } \multicolumn{1}{c|}{} & $\boldsymbol{V}$ & $\boldsymbol{F}$ & $\boldsymbol{a}_{\boldsymbol{p}}$ & $\mathbf{Q}$ & $\mathbf{E}(\mathbf{Q}) / \boldsymbol{\sigma}(\mathbf{R a})$ & $\mathbf{R a}$ & $\mathbf{E}(\mathbf{R a}) / \boldsymbol{\sigma}(\mathbf{R a})$ & $\boldsymbol{\Delta} \mathbf{c}_{\text {vibration }}$ & $\boldsymbol{\Delta} \mathbf{r}_{\text {vibration }}$ \\
\hline Classical optimum & 240.80 & 0.36 & 2 & 173.37 & & 4.20 & & $\mathbf{2 . 5 7}$ & $\mathbf{- 3 . 6 4}$ \\
\hline Robust optimum & 242.10 & 0.33 & 2 & & $146.02 / 5.53$ & & $3.44 / 0.12$ & 21.08 & $\mathbf{1 5 . 3 2}$ \\
\hline
\end{tabular}

$\Delta \mathrm{c}_{\text {vibration }}$ : margins between obtained optima and classical boundaries of feasible domain,

$\Delta r_{\text {vibration }}$ : margins between obtained optima and robust boundaries of feasible domain,

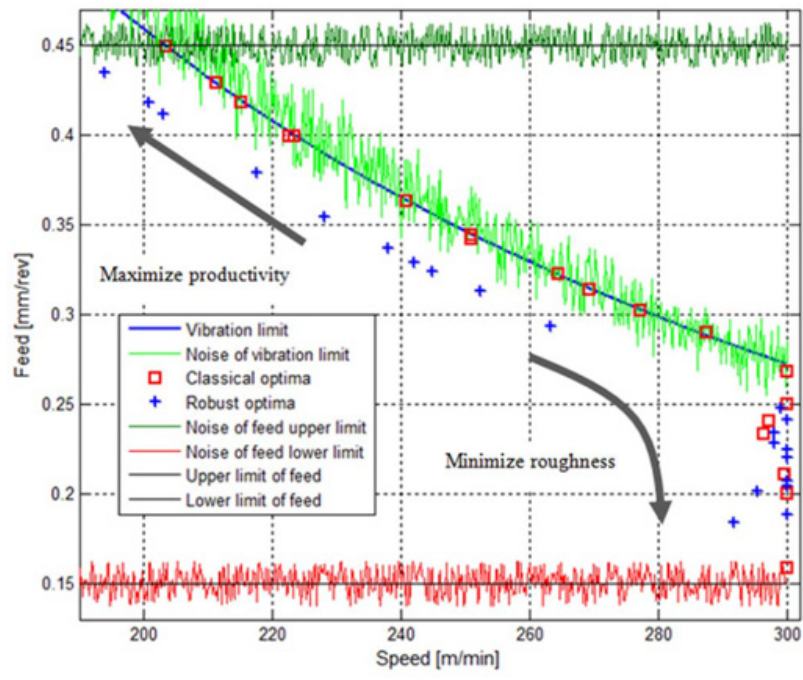

Figure 6. Representation of "classical" and "robust" solutions for $\left(a_{p}=2 \mathrm{~mm}\right)$ respectively in the "classical" and "robust" feasible spaces.

$A_{z \max }$ and maximize productivity (roughing). But, it is recommended to work with the minimum values of feed and maximum values of the cutting speed to minimize surface roughness (finishing). The optimum obtained from the Pareto front without consideration of uncertainties (red squares) are within the "classical" feasible domain, In theory, the constraints imposed are complied with a difference $\Delta c_{\text {vibration }}=2.57 \mathrm{~m} / \mathrm{s}^{2}$ (Table 4) compared to the level of maximum acceleration $A_{z \max }$ for the classical optimum of cutting parameters $(240.8 \mathrm{~m} / \mathrm{s}, 0.36 \mathrm{~mm} / \mathrm{rev}$, $2 \mathrm{~mm}$ ).

However, taking into account uncertainties in the formulation of our problem (Eq. (8)) shows that the classical solutions of Pareto front are not reliable or robust because they are outside the feasible new field called "robust space". Thus, the same classical optimum $(240.8 \mathrm{~m} / \mathrm{s}, 0.36 \mathrm{~mm} / \mathrm{rev}, 2 \mathrm{~mm})$ leads to a default $3.64 \mathrm{~m} / \mathrm{s}^{2}$ compared to the level of maximum accelerations imposed. Therefore the actual limitations defined with taking into account the wear and the tool deformations are raped.

With the developed genetic algorithm incorporating Monte Carlo simulations, robust optima that take into account the variability of the cutting parameters have been obtained. Indeed, for the robust optimum $(242.10 \mathrm{~m} / \mathrm{s}$, $0.33 \mathrm{~mm} / \mathrm{tr}, 2 \mathrm{~mm}$ ), the practical limitations (noise on vibration and maximum advance) are complied with a difference $\Delta r_{\text {vibration }}=15.32 \mathrm{~m} / \mathrm{s}^{2}$ (Table 4) relative to the level of the maximum acceleration.

However, a compromise can be made to obtain the robust optima regarding the constraints imposed and

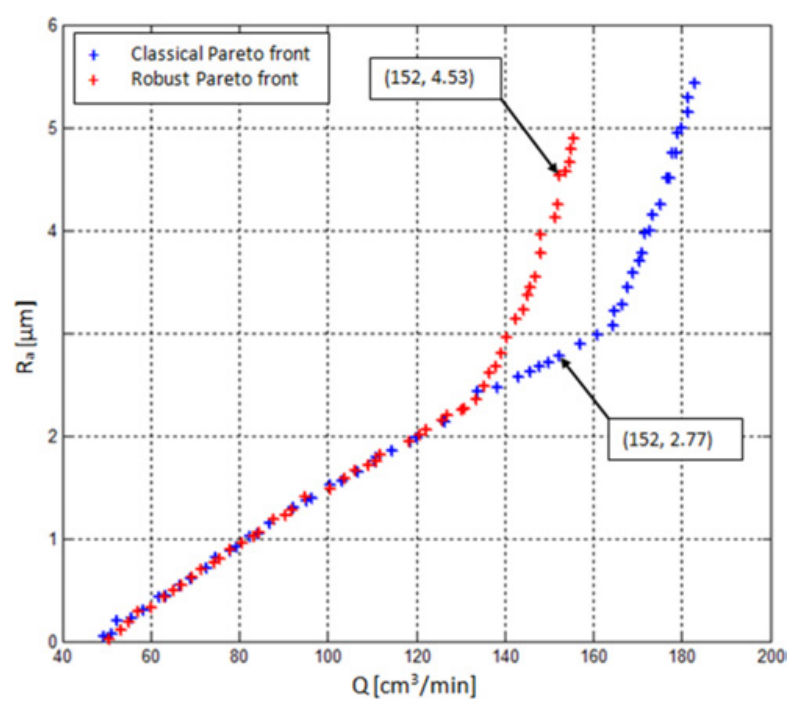

Figure 7. Classical and robust Pareto fronts.

efficient optima regarding the objectives by adjusting the penalty factor $\beta$.

According with the literature, the penalty factor $\beta=3$ in the developed genetic algorithm is chosen. Therefore the robust optima obtained give a good satisfaction for respect of robust constraints imposed and objectives (Fig. 6).

A narrowing of the robust feasible space is noted with the increasing of penalty factor, wear and displacement of the tool. Indeed, an overestimate of the uncertainty interval of uncontrollable parameters can lead to a mediocre efficiency of optima, and the neglect of variability leads to unreliable results.

Taking into account the uncertainties shows us that for a productivity $\left(\mathrm{Q}=152.2 \mathrm{~cm}^{3} / \mathrm{min}\right)$ is accompanied with a real roughness "robust" $4.53 \mu \mathrm{m}$ instead of a theoretical roughness "efficient" $2.77 \mu \mathrm{m}$ (Fig. 7). The robustness and efficiency are two contradictory concepts, where a compromise must be made to satisfy both sides (Table 4).

\section{Conclusion}

Through the design of experiments and multiple linear regressions, we have the relationship between cutting parameters and the variables of interest by the substitution models that have helped us to set the objectives and constraints of our robust optimization problem. By introducing the uncertainties inherent in tool wear and its deflection in the developed optimization algorithm, we obtained "robust" optima for the problem raised.

The developed genetic algorithm incorporating the Monte-Carlo simulation has achieved a compromise 
between robustness and efficiency of the solutions well as to approach the reality of the cutting process. Indeed, we observed that efficient "classical" solutions are not reliable, with an overrun of $3.64 \mathrm{~m} / \mathrm{s}^{2}$ above the level of acceleration allowed. Through the robust optimization algorithm with MC-GA algorithm the imposed limitations are respected by a margin of $\mathbf{1 5 . 3 2} \mathbf{m} / \mathbf{s}^{\mathbf{2}}$ compared to the level of accelerations allowed. After comparing the "classical" and "robust" Pareto fronts, we deduced that a robust (realistic) surface roughness with $\mathbf{R a}=\mathbf{4 . 5 3} \boldsymbol{\mu \mathbf { m }}$ but less efficient can be obtained by taking into account the wear and deflection of the cutting tool unlike that provides deterministic and classical optimization with an efficient roughness $\mathbf{R a}=\mathbf{2 . 7 7} \boldsymbol{\mu} \mathbf{m}$ for the same productivity level $Q=152.2 \mathrm{~cm}^{3} / \mathrm{min}$.

\section{References}

[1] SLOCUM, Alexander H. Precision machine design. SME (1992)

[2] NAWARA, L., KOWALSKI, M., et SLADEK, J. The influence of kinematic errors on the profile shapes by means of CMM. CIRP Annals-Manufacturing Technology, (1989), 38, no 1, p. 511-516

[3] SALGADO, MAa, LÓPEZ DE LACALLE, L. N., LAMIKIZ, A., et al. Evaluation of the stiffness chain on the deflection of end-mills under cutting forces. Int. J. Machine Tools and Manufacture (2005), 45, no 6, p. 727-739

[4] DE LACALLE, LN Lâopez et LAMIKIZ, A. (ed.). Machine tools for high performance machining. Springer (2009)

[5] NAKAZAWA, Hiromu et TAKEGUCHI, R. Principles of precision engineering. Oxford: Oxford university press (1994)

[6] KIM, G. M., KIM, B. H., et CHU, C. N. Estimation of cutter deflection and form error in ball-end milling processes. Int. J. Machine Tools and Manufacture (2003), 43, no 9, p. 917-924

[7] SARTORI, S. et ZHANG, G. X. Geometric error measurement and compensation of machines. CIRP Annals-Manufacturing Technology (1995), 44, no 2, p. 599-609

[8] G. Levaillant, M. Dessoly, P.Ghidossi et al. Usinage par enlèvement de copeaux : de la technologie aux applications industrielles. EYROLLES (2005)

[9] SAHALI, M. A., BELAIDI, I., et SERRA, R. Efficient genetic algorithm for multi-objective robust optimization of machining parameters with taking into account uncertainties. IJAMT, (2014), p. $1-12$

[10] LEE, Kwon-Hee et PARK, Gyung-Jin. Robust optimization considering tolerances of design variables. Comp \& Struct, (2001), 79, no 1, p. 77-86

[11] DOLTSINIS, Ioannis et KANG, Zhan. Robust design of structures using optimization methods. CMAME, (2004), 193, no 23, p. 2221-2237
[12] DU, Xiaoping et CHEN, Wei. Towards a better understanding of modeling feasibility robustness in engineering design. JMD, (2000), 122, p. 385

[13] DAS, Indraneel. Robustness optimization for constrained nonlinear programming problems. EO. A35, (2000), 32, no 5, p. 585-618

[14] JURECKA, Florian. Robust design optimization based on metamodeling techniques. Shaker, (2007)

[15] BAUDOUI, Vincent, KLOTZ, Patricia, HIRIARTURRUTY, Jean-Baptiste, et al. LOcal Uncertainty Processing (LOUP) method for multidisciplinary robust design optimization. Structural and Multidisciplinary Optimization, 2012, 46, no 5, p. 711-726

[16] RAMAKRISHNAN, Balaji et RAO, S. S. A robust optimization approach using Taguchi's loss function for solving nonlinear optimization problems. ADA, (1991), 32, no 1, p. 241-248

[17] APLEY, Daniel W., LIU, Jun, et CHEN, Wei. Understanding the effects of model uncertainty in robust design with computer experiments. JMD, (2006), 128, p. 945

[18] LEHMAN, Jeffrey S., SANTNER, Thomas J., et NOTZ, William I. Designing computer experiments to determine robust control variables. SS (2004), 14, no 2, p. 571-590

[19] SUN, Guangyong, LI, Guangyao, GONG, Zhihui, et al. Multiobjective robust optimization method for drawbead design in sheet metal forming. Materials \& Design, 2010, 31, no 4, p. 1917-1929

[20] JEANG, Angus. Robust cutting parameters optimization for production time via computer experiment. AMM, (2011), 35, no 3, p. 1354-1362

[21] LANDERS, Robert G. et ULSOY, A. Galip. Robust machining force control with process compensation (2003)

[22] IVESTER, R. W. et DANAI, K. Intelligent control of machining under modeling uncertainty. CIRP MS, 1996, 25, no 1, p. 73-79

[23] HUANG, YongAn, ZHANG, Xiaoming, et XIONG, Youlun. Finite Element Analysis of Machining ThinWall Parts: Error Prediction and Stability Analysis (2012)

[24] PAIVA, A. P., CAMPOS, P. H., FERREIRA, J. R., et al. A multivariate robust parameter design approach for optimization of AISI 52100 hardened steel turning with wiper mixed ceramic tool. IJRMHM, (2012), 30, no 1, p. 152-163

[25] SCHIMMERLING, Paul, SISSON, Jean-Claude, et ZAIIDI, Ali. Pratique des plans d'expériences. Tec et doc-Lavoisier (1998)

[26] TINSSON, Walter. Plans d'expérience: constructions et analyses statistiques. Springer (2010)

[27] CHIBANE, Hicham, SERRA, Roger, et LEROY, René. Optimisation des paramètres de coupe en tournage

[28] ISO 3685, Tool-Life Testing with Single-Point Turning Tools, ISO 3685:1993(E), International Standard, 2nd ed., Nov 15 (1993) 